\title{
Peripherally inserted central catheter thrombosis incidence and risk factors in cancer patients: a double-center prospective investigation
}

This article was published in the following Dove Press journal:

Therapeutics and Clinical Risk Management

29 January 2015

Number of times this article has been viewed

\section{Yuxiu Liu' \\ Yufang $\mathrm{Gao}^{3}$ \\ Lili Wei ${ }^{3}$ \\ Weifen Chen' \\ Xiaoyan $\mathrm{Ma}^{4}$ \\ Lei Song ${ }^{2}$}

'Oncology Department, The Affiliated Hospital of Qingdao University, Qingdao, People's Republic of China;

2Department of Breast Oncology, The Affiliated Hospital of Qingdao University, Qingdao, People's Republic of China; ${ }^{3}$ Nursing Department, The Affiliated Hospital of Qingdao University, Qingdao, People's Republic of China; ${ }^{4}$ Intensive Care Unit, Shanghai East Hospital, Shanghai, People's Republic of China
Correspondence: Yufang Gao Nol6 jiangsu Road, The Affiliated Hospital of Qingdao University, Shinan District, Qingdao 26600I, People's Republic of China Email gaoyufang_qd@I63.com
Background: Peripherally inserted central catheters (PICCs) are widely used in chemotherapy, but the reported PICC thrombosis incidence varies greatly, and risks of PICC thrombosis are not well defined. This study was to investigate the incidence and risk factors of PICC-related upper extremity vein thrombosis in cancer patients.

Methods: This was a prospective study conducted in two tertiary referral hospitals from May 2010 to February 2013. Cancer patients who were subject to PICC placement were enrolled and checked by Doppler ultrasound weekly for at least 1 month. Univariable and multivariable logistic regression analyses were applied for identification of risk factors.

Results: Three hundred and eleven cancer patients were enrolled in the study. One hundred and sixty (51.4\%) developed PICC thrombosis, of which 87 (54.4\%) cases were symptomatic. The mean time interval from PICC insertion to thrombosis onset was $11.04 \pm 5.538$ days. The univariable logistic regression analysis showed that complications (odds ratio [OR] 1.686, $P=0.032$ ), less activity (OR $1.476, P=0.006$ ), obesity (OR 3.148, $P=0.000$ ), and chemotherapy history (OR 3.405, $P=0.030$ ) were associated with PICC thrombosis. Multivariate analysis showed that less activity (OR 9.583, $P=0.000$ ) and obesity (OR 3.466, $P=0.014$ ) were significantly associated with PICC thrombosis.

Conclusions: The incidence of PICC thrombosis is relatively high, and nearly half are asymptomatic. Less activity and obesity are risk factors of PICC-related thrombosis.

Keywords: PICC, complication, clinical study, catheter-related thrombosis, upper extremity vein thrombosis

\section{Introduction}

A peripherally inserted central catheter (PICC) is a central vascular access inserted through the peripheral vein of the arm. In recent years, the use of PICCs has increased significantly in the People's Republic of China, especially for cancer patients receiving chemotherapy. For cancer patients, PICCs afford many advantages: eg, avoiding central venous catheter (CVC) placement-associated mechanical complications like pneumothorax, hemorrhage, and facilitating transitions from hospital to intermediate care settings and home for intermittent chemotherapy. ${ }^{1,2}$ Moreover, the professional nurse PICC teams have made their use more convenient and accessible in oncology departments.

Despite these advantages, the high rate of complications of PICCs has raised concerns of clinical medical staff. Catheter-related bloodstream infection and thrombosis are two of the most serious complications of PICCs. Compared with catheter-related bloodstream infection, the studies on thrombosis associated with PICCs are relatively fewer, and many are retrospective in design. What is more, there is less focus on cancer patients' 
PICC-related thrombosis. The consequences of PICC thrombosis should not be omitted. It can result in catheter removal, interruptions in treatment, and acute, life-threatening events such as pulmonary embolism and posthrombotic syndrome. ${ }^{3}$

The reported rates of thrombosis associated with PICC vary greatly. Symptomatic PICC-related thrombosis has been reported to be $1 \%-25.7 \%,{ }^{4-6}$ while the rate of asymptomatic thrombosis has been reported to be up to $35 \%-71.9 \%{ }^{7,8}$ The large difference may lie in study design. As many studies use a retrospective design, evaluating only symptomatic patients or using different diagnostic techniques, the actual rate of thrombosis associated with PICC is still not well defined.

Risk factors of catheter-associated thrombosis can be categorized according to three types: catheter factors such as catheter size and type, tip location, insertion site, numbers of venous punctures, and catheter dwell time; ${ }^{9}$ patient-related factors such as malignancy, recent trauma or surgery, history of venous thromboembolism, older age, and renal failure; and medication factors such as chemotherapy, kinds of infusion liquids, and use of prophylactic anticoagulant. But the risk factors reported in different studies differ greatly.

Although venous angiography is considered to be the gold standard for diagnosis of thrombosis, ultrasound (US) is more often used in diagnosing upper extremity venous thrombosis for its noninvasive, safe, fast, and low-cost characteristics making it the ideal method. US has been widely used in diagnosis of lower limb deep venous thrombosis. As for thrombosis, Doppler US (DU) with compression was reported to have a pooled sensitivity of $91 \%$ and specificity of $93 \%$ in diagnosing upper extremity venous thrombosis in a meta-analysis. ${ }^{10}$ Our study aimed to investigate the incidence of PICC-related thrombosis and identify risk factors using a big sample, multicenter, prospective study via DU examination in cancer patients.

\section{Patients and methods Study population and data collection}

Our data were collected in two tertiary referral hospitals from May 2010 to February 2013. We got the approvals from the Institutional Review Boards at the two hospitals, and the study was in adherence with the Declaration of Helsinki. All enrolled cancer patients provided written informed consent before participation. Identical study protocol and data collection forms were used at both centers. All study data on the patients, including background data and follow-up data, were provided by the study centers in the form of a completed case report form.

Cancer patients who had a PICC placed and who were cared for once a week in one of the two tertiary referral hospitals were approached to participate in the study. The inclusion criteria were as follows: 1) the PICC was placed by PICC specialist nurses, was cared for once a week in the same center and could be followed up; 2) patients were diagnosed with malignancy and needed chemotherapy by PICC; and 3) patients were aged $\geq 18$ years. Exclusion criteria included the following: 1) patients could not come to the appointed department for PICC care every week; 2) patients refused to give written informed consent; 3 ) patients suffered from hematologic diseases; and 4) patients had another venous catheter in the same side as the PICC.

The data collection case report form contained the following items: 1) patients' demographics, containing name, admission number, age, and sex; 2) clinical data, containing clinical diagnosis, comorbidities, thrombosis history, surgical history, trauma history, family medical history, smoking history, CVC history, and activity amount of PICC arm; 3) catheter insertion records, containing insertion date, operator, indication for insertion, number of insertion attempts, vein and arm of insertion, the type of PICC (lumens, gauge, brand), and location of catheter tip; 4) laboratory results, such as routine blood test results, blood lipid result, and blood coagulation results; and 5) DU follow-up records, including the vein monitored, DU results, diagnostic criteria, relevant symptoms, and signs of thrombosis. The data for 1), 2), 3), and 4) were obtained by nursing team records or the hospital information system, and DU data were collected by the authors.

\section{Catheter insertion, DU examination, and follow-up}

All the PICCs were inserted by professional PICC nurses in a separated sterile ward to ensure all the operations were carried out in a sterile environment. Portable US is routinely performed before PICC placement to identify a suitable vein for insertion, and the basilic or cephalic veins were chosen according to the blood vessel assessment result. After PICC insertion, chest radiography was routinely carried out to identify the catheter tip position.

The PICCs were flushed with $10 \mathrm{~mL}$ saline after placement, after each use, and once a week between chemotherapy. The catheter entrance site was covered with dressing and changed every week with thorough skin disinfection. DU checks were performed weekly by the vascular nursing team and a specialist with color Doppler flow imaging certification, who knew nothing about patients' conditions. The follow-up time was for at least 1 month. Some patients were followed for a little longer than 1 month. After every US examination, the clinical symptoms of thrombosis were also documented when present. The veins we examined included cephalic 
veins, basilic veins, axillary veins, innominate veins, and subclavian veins. We also routinely checked the internal jugular veins to identify if PICC malpositions existed.

All PICCs used for our study were 4F single lumen (Bard Groshong PICC, Bard Access Systems, Salt Lake City, UT, USA, and BD open-ended PICC). The DU machine used to evaluate veins and diagnose PICC-related thrombosis was a portable one with an $8 \mathrm{MHz}$ linear probe.

\section{DU diagnosis criteria for thrombosis}

We used two-dimensional scanning with compression testing, color Doppler flow and pulsatility, and variation with physiologic maneuvers to detect the existence of thrombosis.

The major diagnostic criteria of color DU were as follows: 1) loss of compression of imaged vein walls when pressure is applied on the skin during real-time imaging; 2) visualization of echogenic material in the vein; 3) blood flow defect in color flow Doppler imaging; 4) loss or reduction of Doppler velocity spectrum changes; 5) reduction or disappearance of pulsatility and variation with physiologic maneuvers such as rapid inspiration.

The first three diagnostic criteria were direct evidence of thrombus and had important clinical value in diagnosis of thrombosis. The last two were indirect evidence of thrombus by altered blood flow patterns and spectral Doppler indirectly deducing the existence of venous obstruction. ${ }^{11,12}$

\section{Statistical analysis}

Descriptive statistics for characterizing the study population and PICC-related thrombosis were used. The differences of PICC thrombosis rate in different groups were evaluated with $\chi^{2}$ tests. We transformed some numeric variables into ordered categorical variables for further analysis. Univariable logistic regression was used to estimate unadjusted odds ratios (ORs) and 95\% confidence intervals for the association between all risk factors and PICC thrombosis. Variables that were statistically significant with $P<0.1$, as well as those that could have clinical meaning based on the medical literature, were retained in the final multivariable model. Multiple logistic regression analysis was then used to build a model of predictors of thrombosis. Analyses were performed using SPSS 17.0 software, and all statistical tests were two-tailed; $P<0.05$ was considered statistically significant.

\section{Results}

\section{General population data}

From May 2010 to February 2013, a total of 346 cancer patients with 346 PICCs were enrolled at first in the two centers of our study. Of the 346 cancer patients, 311 were followed up for at least 1 month, nine PICCs were removed during the first month for discontinuing therapy (six cases) and catheter occlusion (three cases), and the other 26 were lost to follow-up, with a follow-up efficiency of $89.88 \%$. Therefore, our final study cohort included 311 cancer patients with 311 PICCs, 111 (35.69\%) being men and 200 (64.31\%) being women, with an age range of 22-85 years (mean 54.01 years). The follow-up time was 30-35 days. Of the included patients, there were 132 breast cancer cases, 65 lung cancer cases, 13 esophageal cancer cases, 20 rectal cancer cases, three endometrial carcinoma cases, eleven ovarian cancer cases, seven choriocarcinoma cases, one carcinoma of the parotid gland case, two cervical cancer cases, three liver cancer cases, 30 gastric cancer cases, four pancreatic cancer cases, three nasopharyngeal carcinoma cases, one laryngeal carcinoma case, and 16 colon cancer cases. There were more PICCrelated thrombosis in overweight patients and patients doing less activity $(P<0.05)$. The details are listed in Table 1 .

\section{The general situation of PICC thrombosis}

There were 160 cases of thrombosis in our study, and the incidence was 51.4\%. During our DU follow-up, we found that most patients' upper extremity vein blood flows changed after PICC placement, but the degrees were different. Therefore, we classified the different degrees of thrombosis into four classes according to reports in the literature ${ }^{13}$ and consulting US department experts (Figure 1). Among the 160 PICC thrombosis cases, 85 (53.1\%) were Class I, 23 (14.4\%) were Class II, and 52 (32.5\%) were Class III thrombosis.

The mean time interval from PICC insertion to thrombosis onset was $11.04 \pm 5.538$ days. The earliest thrombosis formed on the second day after catheter insertion, and the latest was 35 days after PICC placement in our study (Figure 2).

In all, 87 (54.4\%) cases showed symptoms of PICC thrombosis. The symptoms in our study included pain along the PICC direction, indurations, forearm swelling, upper arm swelling, and patients' perception of swelling (Table 2).

\section{Risk factors of thrombosis}

A total of 17 variables were entered by univariable logistic regression analyses, including, sex, age, complications, operation history, smoking history, activity amount, obesity, oral anticoagulants, chemotherapy history, vein of PICC, puncture position of PICC, PICC adjustment, side of PICC, number of punctures, catheter tip position, PICC brand, and puncture method. Four risk factors (complications, less activity amount, obesity, and chemotherapy history) were associated with PICC 
Table I General demographic characteristics and thrombosis incidence

\begin{tabular}{|c|c|c|c|c|}
\hline Variable & Patients, N (\%) & Patients with thrombosis, $\mathbf{n}(\%)$ & $\chi^{2}$ & $P$-value \\
\hline Sex & & & 0.001 & 0.980 \\
\hline Male & III (35.7) & $57(51.4)$ & & \\
\hline Female & $200(64.3)$ & $103(51.5)$ & & \\
\hline Age range, years & & & 0.178 & 0.915 \\
\hline $18-45$ & $67(2 \mid .5)$ & $36(53.7)$ & & \\
\hline $46-65$ & $183(58.8)$ & $93(50.8)$ & & \\
\hline$>65$ & $61(9.6)$ & $31(50.8)$ & & \\
\hline Comorbidity & & & 3.277 & 0.657 \\
\hline DM & II (3.5) & $7(63.6)$ & & \\
\hline Hypertension & $29(9.3)$ & $17(58.6)$ & & \\
\hline $\mathrm{CHD}$ & $9(2.9)$ & $5(55.6)$ & & \\
\hline Two kinds & $31(10.0)$ & $20(64.5)$ & & \\
\hline Three kinds & $25(8.0)$ & $14(56.0)$ & & \\
\hline No & $210(67.5)$ & $101(48.1)$ & & \\
\hline Operation & & & 0.464 & 0.496 \\
\hline Yes & $244(78.5)$ & $128(52.5)$ & & \\
\hline No & $67(2 \mid .5)$ & $32(47.8)$ & & \\
\hline Smoking history & & & 1.741 & 0.187 \\
\hline Yes & $61(19.6)$ & $36(59.0)$ & & \\
\hline No & $250(80.4)$ & $124(49.6)$ & & \\
\hline Activity amount & & & 11.329 & $0.003^{\mathrm{a}}$ \\
\hline Mild & $105(33.8)$ & $68(64.8)$ & & \\
\hline Moderate & $103(33.1)$ & 45 (43.7) & & \\
\hline Large & $103(33.1)$ & $47(45.6)$ & & \\
\hline BMI & & & 23.438 & $0.000^{\mathrm{a}}$ \\
\hline$\geq 25$ & I 32 (42.4) & $89(67.4)$ & & \\
\hline$<25$ & $179(57.6)$ & 7I (39.7) & & \\
\hline Insertion vein & & & 0.286 & 0.593 \\
\hline Cephalic & $40(12.9)$ & $19(47.5)$ & & \\
\hline Basilic & $27 \mid(87.1)$ & $|4|(52.0)$ & & \\
\hline Insertion arm & & & 0.730 & 0.393 \\
\hline Left & $125(40.2)$ & $68(54.4)$ & & \\
\hline Right & $186(59.8)$ & $92(49.5)$ & & \\
\hline Insertion position & & & 0.219 & 0.873 \\
\hline Above elbow & $12(3.9)$ & $6(50.0)$ & & \\
\hline Elbow & $107(34.4)$ & $57(53.3)$ & & \\
\hline Below elbow & $192(6 \mid .7)$ & $97(50.5)$ & & \\
\hline Tip position & & & $4.44 I$ & 0.109 \\
\hline Upper $2 / 3$ of SVC & $175(56.3)$ & $84(48.0)$ & & \\
\hline Lower I/3 of SVC & $136(43.7)$ & $76(55.9)$ & & \\
\hline Catheter brand & & & 0.024 & 0.876 \\
\hline $\mathrm{BD}$ & $38(12.2)$ & $20(52.6)$ & & \\
\hline Bard & $273(87.8)$ & $140(5 \mid .3)$ & & \\
\hline
\end{tabular}

Note: ${ }^{a} P<0.01$.

Abbreviations: DM, diabetes mellitus; CHD, coronary heart disease; BMI, body mass index; BD, Becton, Dickinson and Company; SVC, superior vena cava.

thrombosis $(P<0.05)$ (Table 3). To further analyze the risk factors for PICC thrombosis, multivariate analysis for the four risk factors was performed. The results of the multivariate analysis showed two factors with statistical significance: less activity and obesity (Table 4).

\section{Discussion}

The PICC-related upper extremity venous thrombosis incidence in our study was higher than most published data (51.4\%). There were several studies reporting higher PICC thrombosis incidence than ours. A prospective study ${ }^{7}$ showed a $61.9 \%$ thrombosis rate in the upper extremity with a 5F PICC by US examination. The latest article published in 2014 reported a $71.9 \%$ thrombosis incidence by US examination at catheter removal or at 28 days. ${ }^{8}$ Contrasted to these studies, most studies with a retrospective design or containing only symptomatic catheter-related thrombosis reported a lower incidence of PICC thrombosis, 

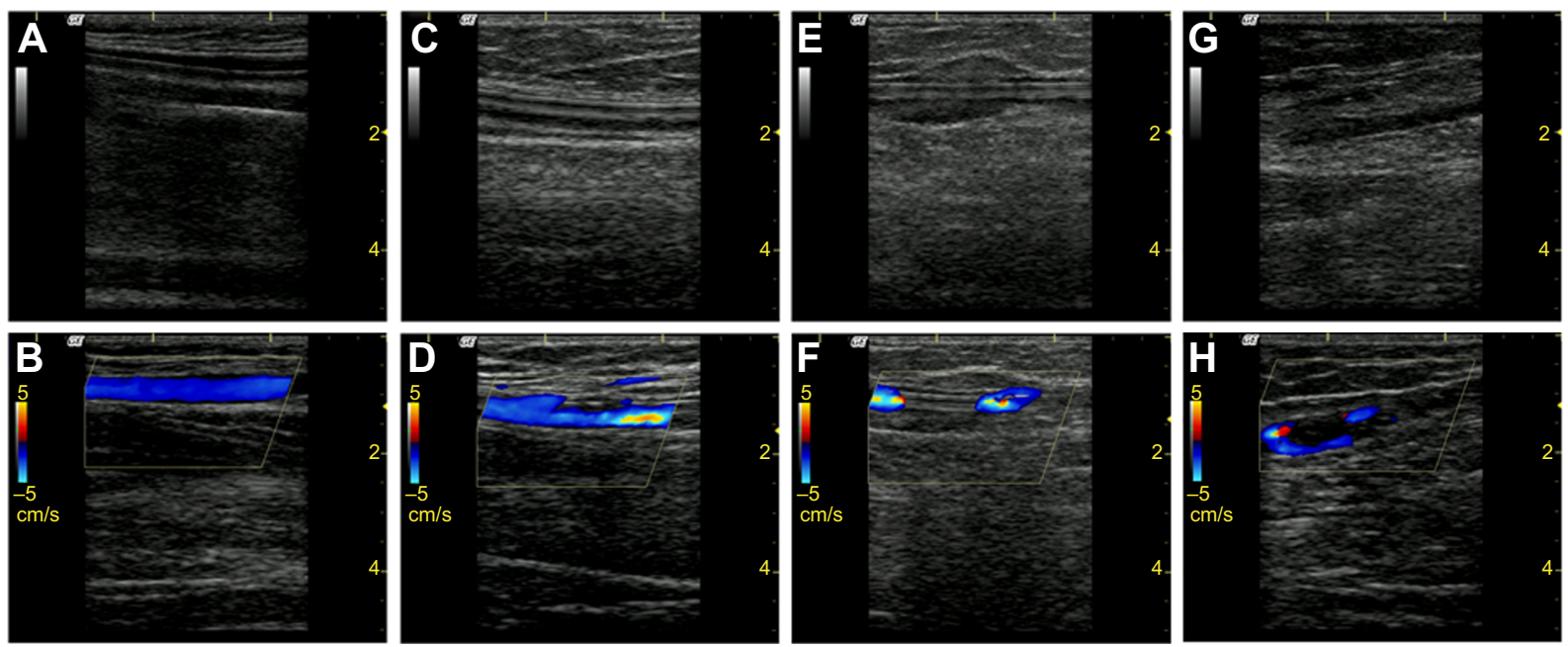

Figure I Two-dimensional and color Doppler flow imaging (CDFI) of different classifications of peripherally inserted central catheter thrombosis. (A) and (B) No thrombosis, complete compressibility of the vein with smooth vascular lumen. (C) and (D) Class I thrombosis, visible small clumps with low echo in lumen of vein and/or catheter outer wall, CDFI showing good blood flow and a vascular stenosis degree of I\%-30\% with slight detectable blood flow. (E) and (F) Class II thrombosis, the presence of thrombosis clot in the vessel lumen and/or catheter outer wall, CDFI showing detectable blood flow and about 31\%-50\% vascular stenosis. (G) and (H) Class III thrombosis, complete blocking thrombosis with a large part of visible fusion of clot, CDFI showing only part signal of blood flow or no flow signals through narrow channel with vessel crosssectional stenosis greater than $50 \%$.

ranging from $1 \%$ to $25.7 \% .^{4-6,14,15}$ The possible causes for the great difference in reported incidence may be study population (cancer patients or general patients), study design (prospective or retrospective), diagnosis measure (US or venography), and screening population (only symptomatic or all). Our study was prospective design in cancer patients and we screened all the included patients by ultrasound. All these reasons may have resulted in a higher PICC-related thrombosis incidence in our study, but our study design also made our study more accurate in evaluating the thrombosis associated with PICCs.
A novel finding in our study was thrombosis associated with PICC classification. During the US examination, we found different grades of PICC thrombosis, which could all be diagnosed as thrombosis according to diagnosing criteria. In order to refine the diagnosis, we classified the PICCrelated thrombosis into four classes, which are illustrated in Figure 1. PICCs in the veins could impact blood flow and the vessels' compressibility, which made it difficulty in diagnosing Class I thrombosis. ${ }^{16}$ In our study, we compared the US results of no thrombosis and Class I thrombosis at different times for different patients and drew the conclusions

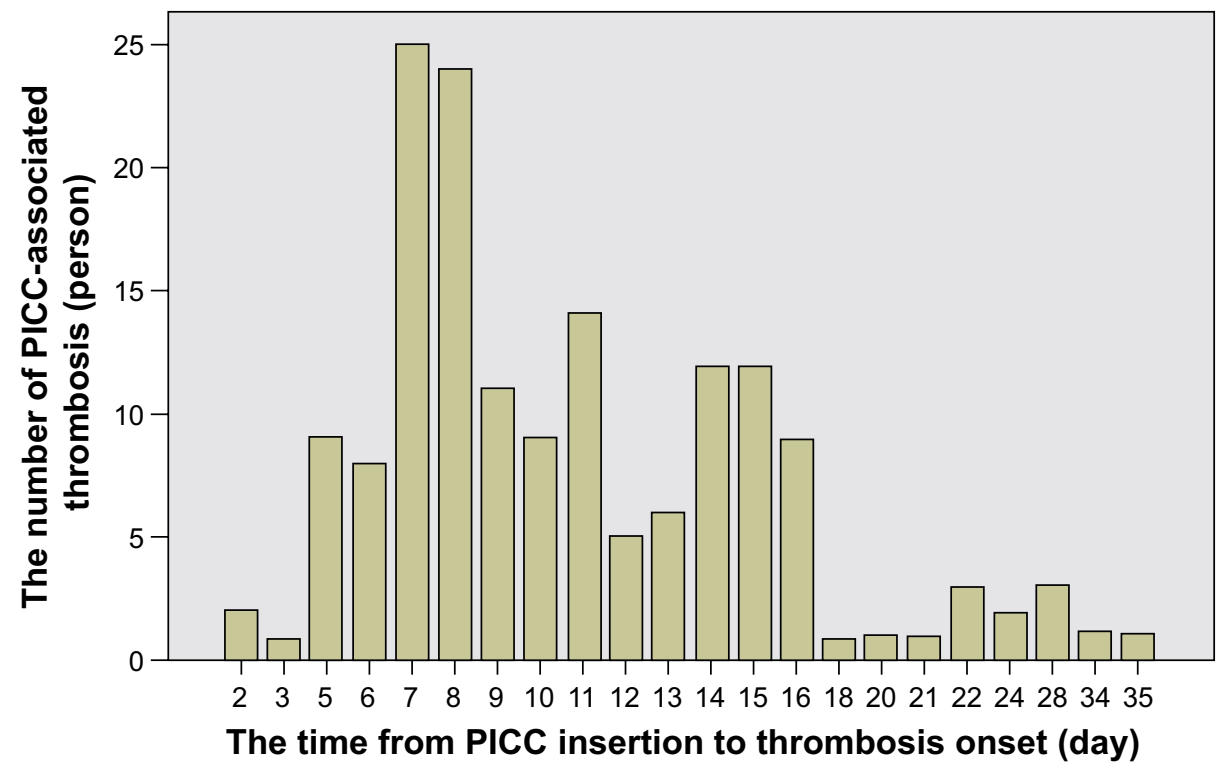

Figure 2 The time distribution between peripherally inserted central catheter (PICC) insertion to the onset of thrombosis. 
Table 2 The symptoms of peripherally inserted central catheter thrombosis in different classifications

\begin{tabular}{lllll}
\hline Symptoms & \multicolumn{2}{l}{ Thrombosis classification } & & Class III, $\mathbf{n}$ \\
\cline { 2 - 5 } & Class I, $\mathbf{n}$ & Class II, $\mathbf{n}$ & 14 & Total, N \\
\hline Phlebitis symptoms, indurations & 7 & 7 & 2 & 28 \\
Swelling of forearm & 1 & 1 & 7 & 4 \\
Swelling of upper extremity & 9 & 2 & 1 & 18 \\
Patients' perception of swelling & 18 & 0 & 9 & 19 \\
Pain or ache & 9 & 0 & 33 & 18 \\
Total & 44 & 10 & & 87 \\
\hline
\end{tabular}

by consensus. From the results in our study, we saw that over half (53.1\%) were Class I thrombosis.

The mean interval of thrombosis from PICC placement to the thrombosis forming in our study was $11.04 \pm 5.538$ days. In Walshe et al's ${ }^{17}$ report, $70 \%$ of thrombus occurred in the first week of insertion, and 30\% developed in the second week, after which no thromboses were diagnosed. $\mathrm{Ng}$ et al ${ }^{18}$ found PICC-related thrombosis forming at a mean time of $12.4 \pm 11$ days. Ong et $\mathrm{al}^{3}$ and King et $\mathrm{al}^{6}$ also reported a mean thrombosis forming time of 15 days. Our finding was similar to the previous literature reports. This finding suggested the significance of prevention in the first half of the month after PICC insertion.

In our study, nearly half of the thrombosis associated with PICC was asymptomatic (45.6\%). This incidence was higher than has been reported before. Luciani et $\mathrm{al}^{16}$ reported a $11.7 \%$ rate of thrombosis associated with CVCs, and $76 \%$ were asymptomatic in cancer patients. In Itkin et al's ${ }^{8}$ study, only $4 \%$ of patients with image-confirmed thrombosis

Table 3 Univariable logistic regression analyses for PICC-related thrombosis

\begin{tabular}{|c|c|c|c|}
\hline Variable & Odds ratio & $95 \% \mathrm{Cl}$ & $P$-value \\
\hline Sex & 1.006 & $0.632-1.600$ & 0.980 \\
\hline Age, years & 0.998 & $0.978-1.018$ & 0.807 \\
\hline Complications & 1.686 & $1.046-2.7 \mid 5$ & $0.032^{\mathrm{a}}$ \\
\hline Operation history & 1.207 & $0.702-2.074$ & 0.496 \\
\hline Smoking history & 1.463 & $0.830-2.580$ & 0.188 \\
\hline Less activity & 1.476 & I.119-1.947 & $0.006^{b}$ \\
\hline Obesity & 3.148 & $1.965-5.044$ & $0.000^{b}$ \\
\hline Oral anticoagulants & 0.758 & $0.433-1.330$ & 0.335 \\
\hline Chemotherapy history & 3.405 & $1.122-10.332$ & $0.030^{\mathrm{a}}$ \\
\hline Vein of PICC & 1.199 & $0.617-2.330$ & 0.593 \\
\hline Puncture position of PICC & 1.067 & $0.720-1.580$ & 0.748 \\
\hline Catheter repositioned & 0.939 & $0.408-2.160$ & 0.883 \\
\hline Side of PICC & 1.219 & $0.774-1.920$ & 0.393 \\
\hline Number of punctures & 0.887 & $0.629-1.249$ & 0.492 \\
\hline Catheter tip position & 0.780 & $0.503-1.208$ & 0.266 \\
\hline PICC brand & 1.056 & $0.535-2.083$ & 0.876 \\
\hline Puncture method & 1.063 & $0.664-1.700$ & 0.799 \\
\hline
\end{tabular}

Notes: ${ }^{a} P<0.05 ;{ }^{b} P<0.01$.

Abbreviations: PICC, peripherally inserted central catheter; $\mathrm{Cl}$, confidence interval. developed clinical symptoms. In our study, the recorded symptoms included phlebitis symptoms, indurations above the puncture point, swollen upper arm, and swollen forearm; what is more, we recorded the patients' consciousness of swelling, which may be the reason that we reported a higher symptomatic thrombosis rate.

The adjusted risk factors in our study by mulitvariable logistic regression analysis were less activity amount and obesity. The findings that obesity and less activity amount were associated with greater risk of PICC thrombosis are in accord with a former published report. ${ }^{19}$ Less activity was defined as activity amount less than activities of daily living (daily activity we perform for self-care such as feeding ourselves, bathing, dressing, grooming etc). Nausea, vomiting, anorexia, fatigue, and other reactions could appear in cancer patients after chemotherapy, which could lead to less activity and more time in bed. What is more, a number of patients deliberately reduced activity of the arm with PICC because of worrying about catheter dislodgment. All these could cause slow blood flow, blood stasis, platelet gathering in catheter wall, and vessel wall damage and eventually form thrombosis. Obesity may add to the difficulty of catheter placement and may be associated with higher blood viscosity, which may increase the risk of thrombosis. In the unadjusted analysis, chemotherapy and complications were also associated with PICC thrombosis in our study. Previously reported factors such as catheter tip position,

Table 4 Multivariable (adjusted) logistic regression analysis of risk factors associated with peripherally inserted central catheter thrombosis

\begin{tabular}{llll}
\hline Variable & Odds ratio & $\mathbf{9 5 \% ~ C l}$ & $P$-value \\
\hline $\begin{array}{l}\text { Complications } \\
\text { (DM, hypertension, CHD) }\end{array}$ & 1.075 & $0.379-3.050$ & $0.89 \mathrm{I}$ \\
Less activity & 9.583 & $3.17 \mathrm{I}-28.962$ & $0.000^{\mathrm{a}}$ \\
Obesity & 3.466 & $1.290-9.31 \mathrm{I}$ & $0.014^{\mathrm{b}}$ \\
Chemotherapy history & 2.107 & $0.594-7.48 \mathrm{I}$ & 0.249 \\
\hline
\end{tabular}

Notes: ${ }^{\mathrm{P}} \mathrm{P}<0.0$ I; ${ }^{\mathrm{b}} \mathrm{P}<0.05$.

Abbreviations: $\mathrm{Cl}$, confidence interval; DM, diabetes mellitus; $\mathrm{CHD}$, coronary heart disease. 
more insertion attempts, and catheter repositioning were not found to have any correlation with thrombosis in our research, which may result from our specialized nursing infusion team for PICC. The impact of certain technical variables that previously potentiated line-related thrombosis was not found in our study.

Compared with previous studies, our study had some advantages. First, our study was a prospective design. We examined the upper-extremity thrombosis 4-6 times by ultrasound during our follow-up time, which made the incidence of PICC thrombosis more accurate. Second, our sample size was larger than most other studies using a prospective design, and our study was done in two centers. Last, we refined the classification of PICC thrombosis, which was an innovation in our study. But there were still limitations in our study. As our US examination was done every week and the follow-up time was only 1 month, the average thrombosis forming time was not exact. In the future, we ought to prolong the follow-up time and increase the frequency of US examination. What is more, we did not do further exploration on the sensitivity and specificity of US diagnosis on thrombosis with PICC insertion, especially for the Class I thrombosis. That will be the direction of our study in the future. Lastly, there were some subjective factors in evaluating the risk factors in our study, and we did not do further research on the consequences of PICC thrombosis. There is still a lot of work to do on PICC-related thrombosis.

\section{Conclusion}

In conclusion, the incidence of upper extremity deep venous thrombosis associated with PICC was higher in cancer patients, with nearly half asymptomatic, which requires further attention. The mean forming time of PICC thrombosis was within 2 weeks, indicating that the prevention of PICCrelated thrombosis in the first 2 weeks after PICC insertion is essential. Our study found that less activity and obesity were key risk factors for PICC thrombosis. This finding highlighted the importance of exercising the arm with PICC in cancer patients.

\section{Acknowledgments}

We acknowledge the PICC team nurses in our two research centers. This work was supported by a medical science and technology development project for Shandong Province (2009 HZ024).

\section{Disclosure}

The authors report no conflicts of interest in this work.

\section{References}

1. Chopra V, Ratz D, Kuhn L, Lopus T, Chenoweth C, Krein S. PICCassociated bloodstream infections: prevalence, patterns, and predictors. Am J Med. 2014;127(4):319-328.

2. Griffiths VR, Philpot P. Peripherally inserted central catheters (PICCs): do they have a role in the care of the critically ill patient? Intensive Crit Care Nurs. 2002;18(1):37-47.

3. Ong B, Gibbs H, Catchpole I, Hetherington R, Harper J. Peripherally inserted central catheters and upper extremity deep vein thrombosis. Australas Radiol. 2006;50(5):451-454.

4. Chemaly RF, de Parres JB, Rehm SJ, et al. Venous thrombosis associated with peripherally inserted central catheters: a retrospective analysis of the Cleveland Clinic experience. Clin Infect Dis. 2002;34(9): $1179-1183$.

5. Cortelezzia A, Fracchiolla NS, Maisonneuve P, et al. Central venous catheter-related complications in patients with hematological malignancies: a retrospective analysis of risk factors and prophylactic measures. Leuk Lymphoma. 2003;44(9):1495-1501.

6. King MM, Rasnake MS, Rodriguez RG, Riley NJ, Stamm JA. Peripherally inserted central venous catheter-associated thrombosis: retrospective analysis of clinical risk factors in adult patients. South Med J. 2006;99(10):1073-1077.

7. Paauw JD, Borders H, Ingalls N, et al. The incidence of PICC lineassociated thrombosis with and without the use of prophylactic anticoagulants. JPEN J Parenter Enteral Nutr. 2008;32(4):443-447.

8. Itkin M, Mondshein JI, Stavropoulos SW, Shlansky-Goldberg RD, Soulen MC, Trerotola SO. Peripherally inserted central catheter thrombosis - reverse tapered versus nontapered catheters: a randomized controlled study. J Vasc Interv Radiol. 2014;25(1):85-91.

9. Gallieni M, Pittiruti M, Biffi R. Vascular access in oncology patients CA Cancer J Clin. 2008;58(6):323-346.

10. Chin EE, Zimmerman PT, Grant EG. Sonographic evaluation of upper extremity deep venous thrombosis. J Ultrasound Med. 2005; 24(6):829-838.

11. Di Nisio M, Van Sluis GL, Bossuyt PM, Buller HR, Porreca E, Rutjes AW. Accuracy of diagnostic tests for clinically suspected upper extremity deep vein thrombosis: a systematic review. J Thromb Haemost. 2010;8(4):684-692.

12. Chin EE, Zimmerman PT, Grant EG. Sonographic evaluation of upper extremity deep venous thrombosis. J Ultrasound Med. 2005;24(6): 829-838; quiz 839-840.

13. Zhao HS, Cheng T, Song YX, Ye YF, Min QF. Significance of regular follow up using color ultrasonography to detect thrombosis in deep veins after tube placing. Chinese J Med ultras (Electronic Version). 2007;4(6):348-350.

14. Aw A, Carrier M, Koczerginski J, McDiarmid S, Tay J. Incidence and predictive factors of symptomatic thrombosis related to peripherally inserted central catheters in chemotherapy patients. Thromb Res. 2012;130(3):323-326.

15. Loughran SC, Borzatta M. Peripherally inserted central catheters: a report of 2,506 catheter days. JPEN J Parenter Enteral Nutr. 1995; 19(2):133-136.

16. Luciani A, Clement O, Halimi P, et al. Catheter-related upper extremity deep venous thrombosis in cancer patients: a prospective study based on Doppler US. Radiology. 2001;220(3):655-660.

17. Walshe LJ, Malak SF, Eagan J, Sepkowitz KA. Complication rates among cancer patients with peripherally inserted central catheters J Clin Oncol. 2002;20(15):3276-3281.

18. Ng PK, Ault MJ, Ellrodt AG, Maldonado L. Peripherally inserted central catheters in general medicine. Mayo Clin Proc. 1997;72(3):225-233.

19. Yi XL, Chen J, Li J, et al. Risk factors associated with PICC-related upper extremity venous thrombosis in cancer patients. J Clin Nurs. 2014;23(5-6):837-843. 


\section{Publish your work in this journal}

Therapeutics and Clinical Risk Management is an international, peerreviewed journal of clinical therapeutics and risk management, focusing on concise rapid reporting of clinical studies in all therapeutic areas, outcomes, safety, and programs for the effective, safe, and sustained use of medicines. This journal is indexed on PubMed Central, CAS,

EMBase, Scopus and the Elsevier Bibliographic databases. The manuscript management system is completely online and includes a very quick and fair peer-review system, which is all easy to use. Visit http://www.dovepress.com/testimonials.php to read real quotes from published authors.

Submit your manuscript here: http://www.dovepress.com/therapeutics-and-clinical-risk-management-journal 